\title{
Existence of nonoscillatory solutions to second-order nonlinear neutral difference equations
}

\author{
Yazhou Tian ${ }^{a, b}$, Yuanli Cai ${ }^{a}$, Tongxing $\mathrm{Li}^{\mathrm{b}, *}$ \\ ${ }^{a}$ School of Electronic and Information Engineering, Xi'an Jiaotong University, Xi'an, Shaanxi 710049, P. R. China. \\ ${ }^{b}$ Qingdao Technological University, Feixian, Shandong 273400, P. R. China.
}

Communicated by Martin Bohner

\section{Abstract}

We study a class of second-order neutral delay difference equations with positive and negative coefficients

$$
\Delta\left(r_{n}\left(\Delta\left(x_{n}+p x_{n-m}\right)\right)\right)+p_{n} f\left(x_{n-k}\right)-q_{n} g\left(x_{n-l}\right)=0, \quad n=n_{0}, n_{0}+1, \ldots,
$$

where $p \in R, m, k, l, n_{0} \in N, p_{n}, q_{n}, r_{n} \in R^{+}, f, g \in C(R, R)$ with $x f(x)>0$ and $x g(x)>0(x \neq 0)$. Some sufficient conditions for the existence of a nonoscillatory solution of the studied equation expressed in terms of $\sum^{\infty} R_{n} p_{n}<\infty$ and $\sum^{\infty} R_{n} q_{n}<\infty$ are obtained, where $R_{n}=\sum_{s=n_{0}}^{n} \frac{1}{r_{s}}, n \geq n_{0}$. (C)2015 All rights reserved.

Keywords: Nonoscillatory solution, neutral delay difference equation, second-order, positive and negative coefficients.

$2010 M S C: 34 \mathrm{~B} 15,34 \mathrm{~B} 25$.

\section{Introduction}

This paper is concerned with a second-order neutral delay difference equation with positive and negative coefficients

$$
\Delta\left(r_{n}\left(\Delta\left(x_{n}+p x_{n-m}\right)\right)\right)+p_{n} f\left(x_{n-k}\right)-q_{n} g\left(x_{n-l}\right)=0, \quad n=n_{0}, n_{0}+1, \ldots,
$$

where $\Delta$ stands for the forward difference operator, $\Delta x_{n}=x_{n+1}-x_{n}, p \in R, m, k, l, n_{0} \in N, p_{n}, q_{n}, r_{n} \in$ $R^{+}, f, g \in C(R, R), x f(x)>0$, and $x g(x)>0$ for all $x \neq 0$. Throughout, we suppose that the following assumptions are satisfied.

\footnotetext{
*Corresponding author

Email addresses: tianyazhou369@163.com (Yazhou Tian), ylicai@mail.xjtu.edu.cn (Yuanli Cai), litongx2007@163.com (Tongxing Li)
} 
$\left(H_{1}\right) f$ and $g$ satisfy local Lipchitz conditions, Lipchitz constants are denoted by $L_{f}(A)$ and $L_{g}(A)$, where $A$ is the domain that $f$ and $g$ are defined;

$\left(H_{2}\right) R_{n}=\sum_{s=n_{0}}^{n} \frac{1}{r_{s}}, n \geq n_{0}, \sum^{\infty} R_{s} p_{s}<\infty$, and $\sum^{\infty} R_{s} q_{s}<\infty$.

In recent years, there has been an increasing interest in studying the oscillatory and nonoscillatory behavior of various classes of differential, difference, and dynamic equations; see, for instance, the monographs [1, 2, papers [3, 4, 5, 6, 7, 8, 9, 10, 11, 12, 13, and the references cited therein. Candan [3] investigated a higher-order nonlinear neutral differential equation

$$
\left[r(t)(x(t)+P(t) x(t-\tau))^{(n-1)}\right]^{\prime}+(-1)^{n}\left[Q_{1}(t) g_{1}\left(x\left(t-\sigma_{1}\right)\right)-Q_{2}(t) g_{2}\left(x\left(t-\sigma_{2}\right)\right)-f(t)\right]=0,
$$

where $t \geq t_{0}, n \geq 2$ is an integer, $r \in C\left(\left[t_{0}, \infty\right), R^{+}\right), P, f \in C\left(\left[t_{0}, \infty\right), R\right), Q_{i} \in C\left(\left[t_{0}, \infty\right), R^{+}\right), i=1,2$, and $g_{i} \in C(R, R), i=1,2$, satisfy the local Lipschitz condition with $x g_{i}(x)>0, i=1,2$ for $x \neq 0$. Using the Banach contraction principle, the author obtained some sufficient conditions for the existence of nonoscillatory solutions to (1.2). Cheng [6] studied the existence of nonoscillatory solution of a second-order linear neutral difference equation

$$
\Delta^{2}\left(x_{n}+p x_{n-m}\right)+p_{n} x_{n-k}-q_{n} x_{n-l}=0, \quad n=n_{0}, n_{0}+1, \ldots,
$$

where $p \in R, m, k, l, n_{0} \in N, p_{n}, q_{n} \in R^{+}$, and some other special cases of equation (1.3) were considered by Li et al. [9] and Zhang and Zhou [13. In particular, Cheng [6] established the following result.

Theorem 1.1 (See [6, Theorem 1]). Suppose that $p \neq-1, \sum^{\infty} s p_{s}<\infty$, and $\sum^{\infty} s q_{s}<\infty$. Then equation (1.3) has a nonoscillatory solution.

To the best of our knowledge, there are few results for second-order nonlinear difference equations with positive and negative coefficients. Motivated by the ideas exploited in [3, 6], we obtain the global results (with respect to $p$ ), which are some sufficient conditions for the existence of a nonoscillatory solution of (1.1) for $p \neq-1$. The results obtained extend those reported in [6]. An example is considered to illustrate the possible applications.

\section{Main results}

Theorem 2.1. Assume that $p \neq-1$ and conditions $\left(H_{1}\right)$ and $\left(H_{2}\right)$ are satisfied. Then (1.1) has a bounded nonoscillatory solution.

Proof. The proof of Theorem 2.1 will be divided into five cases, depending on the five different ranges of the parameter $p$. Let $l_{n_{0}}^{\infty}$ be the Banach space which is composed of all bounded real sequences $x=\left\{x_{n}\right\}_{n=n_{0}}^{\infty}$ with the norm $\|x\|=\sup _{n \geq n_{0}}\left|x_{n}\right|$.

Case 1. $p=1$. By $\left(H_{1}\right)$ and $\left(H_{2}\right)$, one can choose an $n_{*} \geq n_{0}+\max \{m, k, l\}$ sufficiently large such that, for all $n \geq n_{*}$,

$$
\begin{aligned}
& \sum_{u=n}^{\infty}\left(R_{u}-R_{n-1}\right) p_{u} \leq \frac{1}{\alpha}, \\
& \sum_{u=n}^{\infty}\left(R_{u}-R_{n-1}\right) q_{u} \leq \frac{1}{\beta}, \\
& \sum_{u=n}^{\infty}\left(R_{u}-R_{n-1}\right)\left(p_{u}+q_{u}\right)<\min \left\{\frac{1}{L}, \frac{1}{\alpha}+\frac{1}{\beta}\right\},
\end{aligned}
$$

where $\alpha=\max _{1 \leq x \leq 3}\{f(x)\}, \beta=\max _{1 \leq x \leq 3}\{g(x)\}$, and $L=\max \left\{L_{f}([1,3]), L_{g}([1,3])\right\}$.

We define a bounded, closed, and convex subset $S$ in $l_{n_{0}}^{\infty}$ by

$$
S=\left\{x=\left\{x_{n}\right\} \in l_{n_{0}}^{\infty}: 1 \leq x_{n} \leq 3, n \geq n_{0}\right\} .
$$


Consider the operator $T: S \rightarrow l_{n_{0}}^{\infty}$ defined by

$$
(T x)_{n}=\left\{\begin{array}{l}
2-\sum_{j=1}^{\infty} \sum_{s=n+(2 j-1) m}^{n+2 j m}\left(\frac{1}{r_{s}} \sum_{u=s}^{\infty}\left(p_{u} f\left(x_{u-k}\right)-q_{u} g\left(x_{u-l}\right)\right)\right), \quad n \geq n_{*}, \\
(T x)_{n_{*}}, \quad n_{0} \leq n \leq n_{*} .
\end{array}\right.
$$

Clearly, $T x_{n}$ is a real sequence. It is not difficult to show that $T$ is a continuous mapping on $S$. For every $x=\left\{x_{n}\right\} \in S$ and $n \geq n_{*}$, we obtain

$$
\begin{aligned}
(T x)_{n} & \leq 2+\sum_{j=1}^{\infty}\left[\sum_{s=n+(2 j-1) m}^{n+2 j m} \frac{1}{r_{s}} \sum_{u=s}^{\infty} q_{u} g\left(x_{u-l}\right)+\sum_{s=n+(2 j-2) m}^{n+(2 j-1) m} \frac{1}{r_{s}} \sum_{u=s}^{\infty} q_{u} g\left(x_{u-l}\right)\right] \\
& =2+\sum_{s=n}^{\infty} \frac{1}{r_{s}} \sum_{u=s}^{\infty} q_{u} g\left(x_{u-l}\right)=2+\sum_{u=n}^{\infty} \sum_{s=n}^{u} \frac{1}{r_{s}} q_{u} g\left(x_{u-l}\right) \\
& =2+\sum_{u=n}^{\infty}\left(R_{u}-R_{n-1}\right) q_{u} g\left(x_{u-l}\right) \leq 2+\beta \sum_{u=n}^{\infty}\left(R_{u}-R_{n-1}\right) q_{u} \leq 3 .
\end{aligned}
$$

On the other hand, we have

$$
\begin{aligned}
(T x)_{n} & \geq 2-\sum_{j=1}^{\infty}\left[\sum_{s=n+(2 j-1) m}^{n+2 j m} \frac{1}{r_{s}} \sum_{u=s}^{\infty} p_{u} f\left(x_{u-k}\right)+\sum_{s=n+(2 j-2) m}^{n+(2 j-1) m} \frac{1}{r_{s}} \sum_{u=s}^{\infty} p_{u} f\left(x_{u-k}\right)\right] \\
& =2-\sum_{s=n}^{\infty} \frac{1}{r_{s}} \sum_{u=s}^{\infty} p_{u} f\left(x_{u-k}\right)=2-\sum_{u=n}^{\infty} \sum_{s=n}^{u} \frac{1}{r_{s}} p_{u} f\left(x_{u-k}\right) \\
& =2-\sum_{u=n}^{\infty}\left(R_{u}-R_{n-1}\right) p_{u} f\left(x_{u-l}\right) \geq 2-\alpha \sum_{u=n}^{\infty}\left(R_{u}-R_{n-1}\right) p_{u} \geq 1 .
\end{aligned}
$$

Thus, we conclude that $T S \subseteq S$.

Next, we prove that $T$ is a contraction mapping on $S$. As a matter of fact, for every $x, y \in S$ and $n \geq n_{*}$, we get

$$
\begin{aligned}
\left|T x_{n}-T y_{n}\right| & \leq \sum_{j=1}^{\infty} \sum_{s=n+(2 j-1) m}^{n+2 j m}\left(\frac{1}{r_{s}} \sum_{u=s}^{\infty}\left(p_{u}\left|f\left(x_{u-k}\right)-f\left(y_{u-k}\right)\right|+q_{u}\left|g\left(x_{u-l}\right)-g\left(y_{u-l}\right)\right|\right)\right) \\
& \leq L\|x-y\| \sum_{s=n}^{\infty} \frac{1}{r_{s}} \sum_{u=s}^{\infty}\left(p_{u}+q_{u}\right)=L\|x-y\| \sum_{u=n}^{\infty} \sum_{s=n}^{u} \frac{1}{r_{s}}\left(p_{u}+q_{u}\right) \\
& =L\|x-y\| \sum_{u=n}^{\infty}\left(R_{u}-R_{n-1}\right)\left(p_{u}+q_{u}\right)=p_{0}\|x-y\|,
\end{aligned}
$$

which implies that

$$
\|T x-T y\| \leq p_{0}\|x-y\|,
$$

where $p_{0}=L \sum_{u=n}^{\infty}\left(R_{u}-R_{n-1}\right)\left(p_{u}+q_{u}\right)$. Using (2.1), we have $p_{0}<1$, and thus $T$ is a contraction mapping. Consequently, $T$ has a unique fixed $x$ such that $(\overline{T x})_{n}=x_{n}$, that is,

$$
x_{n}=\left\{\begin{array}{l}
2-\sum_{j=1}^{\infty} \sum_{s=n+(2 j-1) m}^{n+2 j m} \frac{1}{r_{s}} \sum_{u=s}^{\infty}\left[p_{u} f\left(x_{u-k}\right)-q_{u} g\left(x_{u-l}\right)\right], \quad n \geq n_{*}, \\
(T x)_{n_{*}}, \quad n_{0} \leq n \leq n_{*} .
\end{array}\right.
$$


Furthermore, we have

$$
\begin{aligned}
x_{n}+x_{n-m} & =4-\sum_{j=1}^{\infty}\left[\sum_{s=n+(2 j-1) m}^{n+2 j m}+\sum_{s=n+(2 j-2) m}^{n+(2 j-1) m}\right] \frac{1}{r_{s}} \sum_{u=s}^{\infty}\left(p_{u} f\left(x_{u-k}\right)-q_{u} g\left(x_{u-l}\right)\right) \\
& =4-\sum_{s=n}^{\infty} \frac{1}{r_{s}} \sum_{u=s}^{\infty}\left(p_{u} f\left(x_{u-k}\right)-q_{u} g\left(x_{u-l}\right)\right) .
\end{aligned}
$$

Therefore,

$$
\Delta\left(r_{n}\left(\Delta\left(x_{n}+x_{n-m}\right)\right)\right)+p_{n} f\left(x_{n-k}\right)-q_{n} g\left(x_{n-l}\right)=0,
$$

and $x_{n}$ is obviously a positive solution of (1.1). This completes the proof of Case 1 .

Case 2. $p \in(0,1)$. By virtue of conditions $\left(H_{1}\right)$ and $\left(H_{2}\right)$, we can choose an $n_{1} \geq n_{0}+\max \{m, k, l\}$ sufficiently large such that

$$
\begin{aligned}
& \sum_{s=n}^{\infty} R_{s} p_{s} \leq \frac{p-\left(1-N_{1}\right)}{\alpha_{1}}, \\
& \sum_{s=n}^{\infty} R_{s} q_{s} \leq \frac{1-p-p N_{1}-M_{1}}{\beta_{1}}, \\
& \sum_{s=n}^{\infty} R_{s}\left(p_{s}+q_{s}\right)<\frac{1-p}{L_{1}}
\end{aligned}
$$

hold for all $n \geq n_{1}$, where $N_{1} \geq M_{1}>0,1-N_{1}<p<\left(1-M_{1}\right) /\left(1+N_{1}\right), \alpha_{1}=\max _{M_{1} \leq x \leq N_{1}}\{f(x)\}$, $\beta_{1}=\max _{M_{1} \leq x \leq N_{1}}\{g(x)\}$, and $L_{1}=\max \left\{L_{f}\left(\left[M_{1}, N_{1}\right]\right), L_{g}\left(\left[M_{1}, N_{1}\right]\right)\right\}$. Set

$$
A_{1}=\left\{x=\left\{x_{n}\right\} \in l_{n_{0}}^{\infty}: M_{1} \leq x_{n} \leq N_{1}, n \geq n_{0}\right\}
$$

Define an operator $T: A_{1} \rightarrow l_{n_{0}}^{\infty}$ by

$$
(T x)_{n}=\left\{\begin{array}{l}
1-p-p x_{n-m}+R_{n-1} \sum_{s=n-1}^{\infty}\left(p_{s} f\left(x_{s-k}\right)-q_{s} g\left(x_{s-l}\right)\right) \\
+\sum_{s=n_{1}}^{n-2} R_{s}\left(p_{s} f\left(x_{s-k}\right)-q_{s} g\left(x_{s-l}\right)\right), \quad n \geq n_{1} \\
(T x)_{n_{1}}, \quad n_{0} \leq n \leq n_{1} .
\end{array}\right.
$$

For every $x \in A_{1}$ and $n \geq n_{1}$, we have

$$
\begin{aligned}
(T x)_{n} & \leq 1-p+\alpha_{1} R_{n-1} \sum_{s=n-1}^{\infty} p_{s}+\alpha_{1} \sum_{s=n_{1}}^{n-2} R_{s} p_{s} \\
& \leq 1-p+\alpha_{1} \sum_{s=n_{1}}^{\infty} R_{s} p_{s} \leq N_{1} .
\end{aligned}
$$

Furthermore, we get

$$
\begin{aligned}
(T x)_{n} & \geq 1-p-p N_{1}-R_{n-1} \sum_{s=n-1}^{\infty} q_{s} g\left(x_{s-l}\right)-\sum_{s=n_{1}}^{n-2} R_{s} q_{s} g\left(x_{s-l}\right) \\
& \geq 1-p-p N_{1}-\beta_{1} \sum_{s=n_{1}}^{\infty} R_{s} q_{s} \geq M_{1}
\end{aligned}
$$


and hence $T A_{1} \subseteq A_{1}$.

Now, for $x, y \in A_{1}$ and $n \geq n_{1}$, we obtain

$$
\begin{aligned}
\left|T x_{n}-T y_{n}\right| \leq & p\left|x_{n-m}-y_{n-m}\right|+R_{n-1} \sum_{s=n-1}^{\infty} p_{s}\left|f\left(x_{s-k}\right)-f\left(y_{s-k}\right)\right| \\
& +R_{n-1} \sum_{s=n-1}^{\infty} q_{s}\left|g\left(x_{s-l}\right)-g\left(y_{s-l}\right)\right|+\sum_{s=n_{1}}^{n-2} R_{s} p_{s}\left|f\left(x_{s-k}\right)-f\left(y_{s-k}\right)\right| \\
& +\sum_{s=n_{1}}^{n-2} R_{s} q_{s}\left|g\left(x_{s-l}\right)-g\left(y_{s-l}\right)\right| \\
\leq & p|| x-y \|+L_{1}|| x-y|| \sum_{s=n_{1}}^{\infty} R_{s}\left(p_{s}+q_{s}\right) \\
= & \hat{q}_{1}|| x-y \|,
\end{aligned}
$$

where $\hat{q}_{1}=p+L_{1} \sum_{s=n_{1}}^{\infty} R_{s}\left(p_{s}+q_{s}\right)<1$ due to 2.2 . This immediately yields

$$
\|T x-T y\| \leq \hat{q}_{1}\|x-y\|,
$$

and so $T$ is a contraction mapping. Consequently, $T$ has a unique fixed $x$, which is obviously a positive solution of (1.1). This completes the proof of Case 2.

Case 3. $p \in(1, \infty)$. From $\left(H_{1}\right)$ and $\left(H_{2}\right)$, one can choose an $n_{2} \geq n_{0}+\max \{m, k, l\}$ sufficiently large such that

$$
\begin{aligned}
& \sum_{s=n}^{\infty} R_{s} p_{s} \leq \frac{1-p\left(1-N_{2}\right)}{\alpha_{2}}, \\
& \sum_{s=n}^{\infty} R_{s} q_{s} \leq \frac{\left(1-M_{2}\right) p-\left(1+N_{2}\right)}{\beta_{2}}, \\
& \sum_{s=n}^{\infty} R_{s}\left(p_{s}+q_{s}\right)<\frac{p-1}{L_{2}}
\end{aligned}
$$

hold for all $n \geq n_{2}$, where $N_{2} \geq M_{2}>0,\left(1-M_{2}\right) p>1+N_{2}, p\left(1-N_{2}\right)<1, \alpha_{2}=\max _{M_{2} \leq x \leq N_{2}}\{f(x)\}$, $\beta_{2}=\max _{M_{2} \leq x \leq N_{2}}\{g(x)\}$, and $L_{2}=\max \left\{L_{f}\left(\left[M_{2}, N_{2}\right]\right), L_{g}\left(\left[M_{2}, N_{2}\right]\right)\right\}$. Set

$$
A_{2}=\left\{x=\left\{x_{n}\right\} \in l_{n_{0}}^{\infty}: M_{2} \leq x_{n} \leq N_{2}, n \geq n_{0}\right\} .
$$

Define an operator $T: A_{2} \rightarrow l_{n_{0}}^{\infty}$ as

$$
(T x)_{n}=\left\{\begin{array}{l}
1-\frac{1}{p}-\frac{1}{p} x_{n+m}+\frac{1}{p} R_{n+m-1} \sum_{s=n+m-1}^{\infty}\left(p_{s} f\left(x_{s-k}\right)-q_{s} g\left(x_{s-l}\right)\right) \\
+\frac{1}{p} \sum_{s=n_{2}}^{n+m-2} R_{s}\left(p_{s} f\left(x_{s-k}\right)-q_{s} g\left(x_{s-l}\right)\right), \quad n \geq n_{2} \\
(T x)_{n_{2}}, \quad n_{0} \leq n \leq n_{2} .
\end{array}\right.
$$

For every $x \in A_{2}$ and $n \geq n_{2}$, we get

$$
\begin{aligned}
(T x)_{n} & \leq 1-\frac{1}{p}+\frac{1}{p} \alpha_{2} R_{n+m-1} \sum_{s=n+m-1}^{\infty} p_{s}+\frac{1}{p} \alpha_{2} \sum_{s=n_{2}}^{n+m-2} R_{s} p_{s} \\
& \leq 1-\frac{1}{p}+\frac{1}{p} \alpha_{2} \sum_{s=n_{2}}^{\infty} R_{s} p_{s} \leq N_{2} .
\end{aligned}
$$


Furthermore, we have

$$
\begin{aligned}
(T x)_{n} & \geq 1-\frac{1}{p}-\frac{1}{p} N_{2}-\frac{1}{p} \beta_{2} R_{n+m-1} \sum_{s=n+m-1}^{\infty} q_{s}-\frac{1}{p} \beta_{2} \sum_{s=n_{2}}^{n+m-2} R_{s} q_{s} \\
& \geq 1-\frac{1}{p}-\frac{1}{p} N_{2}-\frac{1}{p} \beta_{2} \sum_{s=n_{2}}^{\infty} R_{s} q_{s} \geq M_{2},
\end{aligned}
$$

and thus $T A_{2} \subseteq A_{2}$. Since $A_{2}$ is a bounded, closed, and convex subset of $l_{n_{0}}^{\infty}$, we have to prove that $T$ is a contraction mapping on $A_{2}$ to apply the contraction principle.

Now, for $x, y \in A_{2}$ and $n \geq n_{2}$, we obtain

$$
\begin{aligned}
\left|T x_{n}-T y_{n}\right| \leq & \frac{1}{p}\left|x_{n+m}-y_{n+m}\right|+\frac{1}{p} R_{n+m-1} \sum_{s=n+m-1}^{\infty} p_{s}\left|f\left(x_{s-k}\right)-f\left(y_{s-k}\right)\right| \\
& +\frac{1}{p} R_{n+m-1} \sum_{s=n+m-1}^{\infty} q_{s}\left|g\left(x_{s-l}\right)-g\left(y_{s-l}\right)\right|+\frac{1}{p} \sum_{s=n_{2}}^{n+m-2} R_{s} p_{s}\left|f\left(x_{s-k}\right)-f\left(y_{s-k}\right)\right| \\
& +\frac{1}{p} \sum_{s=n_{2}}^{n+m-2} R_{s} q_{s}\left|g\left(x_{s-l}\right)-g\left(y_{s-l}\right)\right| \\
\leq & \frac{1}{p}\|x-y\|+\frac{1}{p} L_{2}|| x-y \| \sum_{s=n_{2}}^{\infty} R_{s}\left(p_{s}+q_{s}\right) \\
= & \hat{q}_{2}|| x-y \|,
\end{aligned}
$$

which yields

$$
\|T x-T y\| \leq \hat{q}_{2}\|x-y\| .
$$

From 2.3), we have $\hat{q}_{2}=1 / p\left(1+L_{2} \Sigma_{s=n_{2}}^{\infty} R_{s}\left(p_{s}+q_{s}\right)\right)<1$. Therefore, $T$ is a contraction mapping. Consequently, $T$ has a unique fixed $x$, which is obviously a positive solution of (1.1). The proof of Case 3 is complete.

Case 4. $p \in(-1,0)$. Combining $\left(H_{1}\right)$ and $\left(H_{2}\right)$, we can choose an $n_{3} \geq n_{0}+\max \{m, k, l\}$ sufficiently large such that

$$
\begin{aligned}
& \sum_{s=n}^{\infty} R_{s} p_{s} \leq \frac{(1+p) N_{3}-(1+p)}{\alpha_{3}}, \\
& \sum_{s=n}^{\infty} R_{s} q_{s} \leq \frac{1+p-M_{3}(1+p)}{\beta_{3}}, \\
& \sum_{s=n}^{\infty} R_{s}\left(p_{s}+q_{s}\right)<\frac{1+p}{L_{3}}
\end{aligned}
$$

hold for all $n \geq n_{3}$, where $M_{3}$ and $N_{3}$ are positive constants satisfying $0<M_{3}<1<N_{3}$, $\alpha_{3}=\max _{M_{3} \leq x \leq N_{3}}\{f(x)\}, \beta_{3}=\max _{M_{3} \leq x \leq N_{3}}\{g(x)\}$, and $L_{3}=\max \left\{L_{f}\left(\left[M_{3}, N_{3}\right]\right), L_{g}\left(\left[M_{3}, N_{3}\right]\right)\right\}$. Set

$$
A_{3}=\left\{x=\left\{x_{n}\right\} \in l_{n_{0}}^{\infty}: M_{3} \leq x_{n} \leq N_{3}, n \geq n_{0}\right\} .
$$

Define an operator $T: A_{3} \rightarrow l_{n_{0}}^{\infty}$ by

$$
(T x)_{n}=\left\{\begin{array}{l}
1+p-p x_{n-m}+R_{n-1} \sum_{s=n-1}^{\infty}\left(p_{s} f\left(x_{s-k}\right)-q_{s} g\left(x_{s-l}\right)\right) \\
+\sum_{s=n_{3}}^{n-2} R_{s}\left(p_{s} f\left(x_{s-k}\right)-q_{s} g\left(x_{s-l}\right)\right), \quad n \geq n_{3} \\
(T x)_{n_{3}}, \quad n_{0} \leq n \leq n_{3} .
\end{array}\right.
$$


For every $x \in A$ and $n \geq n_{3}$, we have

$$
\begin{aligned}
(T x)_{n} & \leq 1+p-p N_{3}+\alpha_{3} R_{n-1} \sum_{s=n-1}^{\infty} p_{s}+\alpha_{3} \sum_{s=n_{3}}^{n-2} R_{s} p_{s} \\
& \leq 1+p-p N_{3}+\alpha_{3} \sum_{s=n_{3}}^{\infty} R_{s} p_{s} \leq N_{3} .
\end{aligned}
$$

Furthermore, we conclude that

$$
\begin{aligned}
(T x)_{n} & \geq 1+p-p M_{3}-R_{n-1} \sum_{s=n-1}^{\infty} q_{s} g\left(x_{s-l}\right)-\sum_{s=n_{3}}^{n-2} R_{s} q_{s} g\left(x_{s-l}\right) \\
& \geq 1+p-p M_{3}-\beta_{3} \sum_{s=n_{3}}^{\infty} R_{s} q_{s} \geq M_{3}
\end{aligned}
$$

and thus $T A_{3} \subseteq A_{3}$.

Next, we prove that $T$ is a contraction mapping on $A_{3}$. In fact, for every $x, y \in A_{3}$ and $n \geq n_{3}$, we have

$$
\begin{aligned}
\left|T x_{n}-T y_{n}\right| \leq & -p\left|x_{n-m}-y_{n-m}\right|+R_{n-1} \sum_{s=n-1}^{\infty} p_{s}\left|f\left(x_{s-k}\right)-f\left(y_{s-k}\right)\right| \\
& +R_{n-1} \sum_{s=n-1}^{\infty} q_{s}\left|g\left(x_{s-l}\right)-g\left(y_{s-l}\right)\right|+\sum_{s=n_{3}}^{n-2} R_{s} p_{s}\left|f\left(x_{s-k}\right)-f\left(y_{s-k}\right)\right| \\
& +\sum_{s=n_{3}}^{n-2} R_{s} q_{s}\left|g\left(x_{s-l}\right)-g\left(y_{s-l}\right)\right| \\
\leq & -p\|x-y\|+L_{3}|| x-y \| \sum_{s=n_{3}}^{\infty} R_{s}\left(p_{s}+q_{s}\right) \\
= & \hat{q}_{3}|| x-y \| .
\end{aligned}
$$

This immediately yields

$$
\|T x-T y\| \leq \hat{q}_{3}\|x-y\|,
$$

where $\hat{q}_{3}=-p+L_{3} \sum_{s=n_{3}}^{\infty} R_{s}\left(p_{s}+q_{s}\right)<1$ due to (2.4), which implies that $T$ is a contraction mapping. Consequently, $T$ has a unique fixed $x$, which is obviously a positive solution of (1.1). This completes the proof of Case 4.

Case 5. $p \in(-\infty,-1)$. From $\left(H_{1}\right)$ and $\left(H_{2}\right)$, one can choose an $n_{4} \geq n_{0}+\max \{m, k, l\}$ sufficiently large such that

$$
\begin{aligned}
& \sum_{s=n}^{\infty} R_{s} p_{s} \leq \frac{-(p+1)\left(N_{4}-1\right)}{\beta_{4}} \\
& \sum_{s=n}^{\infty} R_{s} q_{s} \leq \frac{-(1+p)\left(1-M_{4}\right)}{\alpha_{4}} \\
& \sum_{s=n}^{\infty} R_{s}\left(p_{s}+q_{s}\right)<\frac{-(p+1)}{L_{4}}
\end{aligned}
$$

hold for all $n \geq n_{4}$, where $M_{4}$ and $N_{4}$ are positive constants satisfying $0<M_{4}<1<N_{4}$, $\alpha_{4}=\max _{M_{4} \leq x \leq N_{4}}\{f(x)\}, \beta_{4}=\max _{M_{4} \leq x \leq N_{4}}\{g(x)\}$, and $L_{4}=\max \left\{L_{f}\left(\left[M_{4}, N_{4}\right]\right), L_{g}\left(\left[M_{4}, N_{4}\right]\right)\right\}$. Set

$$
A_{4}=\left\{x=\left\{x_{n}\right\} \in l_{n_{0}}^{\infty}: M_{4} \leq x_{n} \leq N_{4}, n \geq n_{0}\right\} .
$$


Define an operator $T: A_{4} \rightarrow l_{n_{0}}^{\infty}$ as

$$
(T x)_{n}=\left\{\begin{array}{l}
1+\frac{1}{p}-\frac{1}{p} x_{n+m}+\frac{1}{p} R_{n+m-1} \sum_{s=n+m-1}^{\infty}\left(p_{s} f\left(x_{s-k}\right)-q_{s} g\left(x_{s-l}\right)\right) \\
+\frac{1}{p} \sum_{s=n_{4}}^{n+m-2} R_{s}\left(q_{s} f\left(x_{s-k}\right)-q_{s} g\left(x_{s-l}\right)\right), \quad n \geq n_{4}, \\
(T x)_{n_{4}}, \quad n_{0} \leq n \leq n_{4} .
\end{array}\right.
$$

For every $x \in A_{4}$ and $n \geq n_{4}$, we get

$$
\begin{aligned}
(T x)_{n} & \leq 1+\frac{1}{p}-\frac{1}{p} N_{4}-\frac{1}{p} \beta_{4} R_{n+m-1} \sum_{s=n+m-1}^{\infty} q_{s}-\frac{1}{p} \beta_{4} \sum_{s=n_{4}}^{n+m-2} R_{s} q_{s} \\
& \leq 1+\frac{1}{p}-\frac{1}{p} N_{4}-\frac{1}{p} \beta_{4} \sum_{s=n_{4}}^{\infty} R_{s} q_{s} \leq N_{4} .
\end{aligned}
$$

Furthermore, we have

$$
\begin{aligned}
(T x)_{n} & \geq 1+\frac{1}{p}-\frac{1}{p} M_{4}+\frac{1}{p} \alpha_{4} R_{n+m-1} \sum_{s=n+m-1}^{\infty} p_{s}+\frac{1}{p} \alpha_{4} \sum_{s=n_{4}}^{n+m-2} R_{s} p_{s} \\
& \geq 1+\frac{1}{p}-\frac{1}{p} M_{4}+\frac{1}{p} \alpha_{4} \sum_{s=n_{4}}^{\infty} R_{s} q_{s} \geq M_{4},
\end{aligned}
$$

and so $T A_{4} \subseteq A_{4}$. Since $A_{4}$ is a bounded, closed, and convex subset of $l_{n_{0}}^{\infty}$, we have to prove that $T$ is a contraction mapping on $A_{4}$ to apply the contraction principle.

Now, for $x, y \in A_{4}$ and $n \geq n_{4}$, we have

$$
\begin{aligned}
\left|T x_{n}-T y_{n}\right| \leq & -\frac{1}{p}\left|x_{n+m}-y_{n+m}\right|-\frac{1}{p} R_{n+m-1} \sum_{s=n+m-1}^{\infty} p_{s}\left|f\left(x_{s-k}\right)-f\left(y_{s-k}\right)\right| \\
& -\frac{1}{p} R_{n+m-1} \sum_{s=n+m-1}^{\infty} q_{s}\left|g\left(x_{s-l}\right)-g\left(y_{s-l}\right)\right|-\frac{1}{p} \sum_{s=n_{4}}^{n+m-2} R_{s} p_{s}\left|f\left(x_{s-k}\right)-f\left(y_{s-k}\right)\right| \\
& -\frac{1}{p} \sum_{s=n_{4}}^{n+m-2} R_{s} q_{s}\left|g\left(x_{s-l}\right)-g\left(y_{s-l}\right)\right| \\
\leq & -\frac{1}{p}|| x-y \|-\frac{1}{p} L_{4}|| x-y|| \sum_{s=n_{4}}^{\infty} R_{s}\left(p_{s}+q_{s}\right) \\
= & \hat{q}_{4}|| x-y \| .
\end{aligned}
$$

This immediately implies that

$$
\|T x-T y\| \leq \hat{q}_{4}\|x-y\| .
$$

By virtue of 2.5), we get $\hat{q}_{4}=1 / p\left(-1-L_{4} \Sigma_{s=n_{4}}^{\infty} R_{s}\left(p_{s}+q_{s}\right)\right)<1$, which proves that $T$ is a contraction mapping. Consequently, $T$ has a unique fixed $x$, which is obviously a positive solution of (1.1). This completes the proof of Case 5. Therefore, the proof of Theorem 2.1 is complete.

Remark 2.2. One can easily see that Theorem 2.1 includes Theorem 1.1 when $r_{n}=1$ and $f(u)=g(u)=u$. 


\section{Applications}

Example 3.1. Consider a second-order difference equation

$$
\Delta\left(r_{n} \Delta\left(x_{n}+x_{n-1}\right)\right)+p_{n} x_{n-2}-q_{n} x_{n-2}^{3}=0, \quad n=2,3, \ldots,
$$

where $p=1, r_{n}=1 / n, f(x)=x, g(x)=x^{3}$,

$$
p_{n}=\frac{2(n-2)}{(n+2)(n+1) n(n-1)(2 n-3)},
$$

and

$$
q_{n}=\frac{8(n-2)^{3}}{(n+2)(n+1) n(n-1)(2 n-3)^{3}} .
$$

It is easy to verify that

$$
\begin{aligned}
& R_{n}=\sum_{s=2}^{n} \frac{1}{r_{s}}=\sum_{s=2}^{n} s=\frac{1}{2}(n+2)(n-1), \\
& \sum_{n=2}^{\infty} R_{n} p_{n}<\infty, \text { and } \sum_{n=2}^{\infty} R_{n} q_{n}<\infty .
\end{aligned}
$$

Therefore, conditions $\left(H_{1}\right)$ and $\left(H_{2}\right)$ are satisfied. By Theorem 2.1, equation (3.1) has a bounded nonoscillatory solution. As a matter of fact, the sequence $\left\{x_{n}\right\}=\{2+1 / n\}$ is a nonoscillatory solution of (3.1).

\section{Acknowledgements}

This research was supported by the NNSF of China (Grant Nos. 11171178 and 11271225) and the NSF of Shandong Province (ZR2012AL03).

\section{References}

[1] R. P. Agarwal, M. Bohner, S. R. Grace, D. O'Regan, Discrete Oscillation Theory, Hindawi Publishing Corporation, New York, (2005).1

[2] R. P. Agarwal, P. J. Y. Wong, Advanced Topics in Difference Equations, Kluwer Academic Publishers, Dordeecht, (1997). 1

[3] T. Candan, The existence of nonoscillatory solutions of higher order nonlinear neutral equations, Appl. Math. Lett., 25 (2012), 412-416.1 1

[4] T. Candan, R. S. Dahiya, Existence of nonoscillatory solutions of first and second order neutral differential equations with distributed deviating arguments, J. Franklin Inst., 347 (2010), 1309-1316.1.

[5] S. Chen, C. Li, Nonoscillatory solutions of second order nonlinear difference equations, Appl. Math. Comput., 205 (2008), 478-481.1

[6] J. Cheng, Existence of a nonoscillatory solution of a second-order linear neutral difference equation, Appl. Math. Lett., 20 (2007), 892-899.1, 1, 1, 1.1, 1

[7] B. S. Lalli, B. G. Zhang, On existence of positive solutions and bounded oscillations for neutral difference equations, J. Math. Anal. Appl., 166 (1992), 272-287.1

[8] T. Li, Z. Han, S. Sun, D. Yang, Existence of nonoscillatory solutions to second-order neutral delay dynamic equations on time scales, Adv. Difference Equ., 2009 (2009), 10 pages.1

[9] W. T. Li, X. L. Fan, C. k. Zhong, On unbounded positive solutions of second-order difference equations with a singular nonlinear term, J. Math. Anal. Appl., 246 (2000), 80-88.1. 1

[10] H. Peics, Positive solutions of second-order linear difference equation with variable delays, Adv. Difference Equ., 2013 (2013), 12 pages.1

[11] Y. Tian, F. Meng, Existence for nonoscillatory solutions of higher-order nonlinear differential equations, ISRN Math. Anal., 2011 (2011), 9 pages.1.

[12] J. Yan, Existence of oscillatory solutions of forced second order delay differential equations, Appl. Math. Lett., 24 (2011), 1455-1460.1

[13] B. G. Zhang, Y. Zhou, Oscillation and nonoscillation for second-order linear difference equations, Comput. Math. Appl., 39 (2000), 1-7.1.1 